\title{
ACE2 and TMPRSS2 variants and expression as candidates to sex and country differences in COVID-19 severity in Italy
}

Rosanna Asselta, $\mathrm{PhD}^{1,2^{*}}$ Elvezia Maria Paraboschi, $\mathrm{PhD}^{1,2^{*}}$, Alberto Mantovani, $\mathrm{MD}^{1,2,3}$ Stefano Duga, $\mathrm{PhD}^{1,2}$

1 Department of Biomedical Sciences, Humanitas University, Via Rita Levi Montalcini 4, 20090 Pieve Emanuele, Milan, Italy;

2 Humanitas Clinical and Research Center, IRCCS, Via Manzoni 56, 20089 Rozzano, Milan, Italy;

${ }^{3}$ The William Harvey Research Institute, Queen Mary University of London, London EC1M 6BQ, UK.

* These authors contributed equally to this work

Short title: TMPRSS2 and COVID-19 in the Italian population

Key words: SARS-CoV-2, COVID-19, ACE2, TMPRSS2, genetic variants, Italian population

\section{Corresponding author:}

Stefano Duga

Department of Biological Sciences

Humanitas University

Pieve Emanuele, Milan, Italy

Phone: +390282245204

Email: stefano.duga@hunimed.eu 


\section{ABSTRACT}

\section{Background}

As the outbreak of coronavirus disease 2019 (COVID-19) progresses, prognostic markers for early identification of high-risk individuals are an urgent medical need. Italy has the highest rate of SARS-CoV-2 infection, the highest number of deaths, and the highest mortality rate among large countries. Worldwide, a more severe course of COVID-19 is associated with older age, comorbidities, and male sex. Hence, we searched for possible genetic components of the peculiar severity of COVID-19 among Italians, by looking at expression levels and variants in ACE2 and TMPRSS2 genes, which are crucial for viral infection.

\section{Methods}

Exome and SNP array data from a large Italian cohort representative of the country's population were used to compare the burden of rare variants and the frequency of polymorphisms with European and East Asian populations. Moreover, we looked into gene expression databases to check for sex-unbalanced expression.

\section{Results}

While we found no significant evidence that ACE2 is associated with disease severity/sex bias in the Italian population, TMPRSS2 levels and genetic variants proved to be possible candidate disease modulators, contributing to the observed epidemiological data among Italian patients.

\section{Conclusions}

Our analysis suggests a role for TMPRSS2 variants and expression levels in modulating COVID-19 severity, a hypothesis that fosters a rapid experimental validation on large cohorts of patients with different clinical manifestations. 


\section{INTRODUCTION}

As we write, Italy, Europe, and the entire world are facing one of the worst medical emergencies spanning centuries, the coronavirus disease 2019 (COVID-19) pandemia due to infection by SARS-CoV-2 virus. The early identification of risk factors for COVID-19 is an urgent medical need to provide the appropriate support to patients, including access to intensive care units.

Presently, Italy has one of the highest rate of SARS-CoV-2 infection in the world among large countries, with 143 cases per 100,000 people, the highest number of deaths and the highest mortality rate, $10.5 \%$ vs. an average value of $4.6 \%$ (as of March 28th, 2020, data from https://coronavirus.jhu.edu/map.html). These data may have different explanations, including: 1) the number of tests performed, 2) the structure of the population (Italy has the oldest population in Europe) [https://ec.europa.eu/eurostat/data/database], 3) the percentage of smokers, even though no significant association was found between smoking and severity of COVID19 in a very recent study on the Chinese population, $\left.{ }^{1} 4\right)$ the possible existence of a different virus strain, ${ }^{2} 5$ ) the concentration of severe cases in a limited region of the country, potentially overwhelming the available intensive care units, as well as 6) differences in environmental factors (e.g. air pollution). However, there could also be some peculiar genetic characteristics of the Italian population that may have an impact on the susceptibility to viral infection, the disease severity, and the number of patients shedding huge amounts of virus.

What is unquestionable is a more severe course of the disease associated with older age and high number of comorbidities and with the male sex (male:female ratio in case fatality rate among Italians 1.75 , data from the Italian National Institute of Health: https://www.epicentro.iss.it/coronavirus/), a feature shared with the 2003 SARS epidemic and MERS. ${ }^{3-5}$ Indeed, while men and women have similar susceptibility to both SARS-CoV-2 and SARS-CoV, men are more prone to have higher severity and mortality, independently of age. ${ }^{3}$ Among the many possible factors impacting on sexrelated differences in disease manifestations, including the fact that females are known to mount a stronger immune response to viral infections compared to males due to more robust humoral and cellular immune responses, ${ }^{6}$ we decided to center our attention on possible genetic components, with a particular focus on the Italian population. 
It was recently demonstrated that both angiotensin I converting enzyme 2 (ACE2) and the transmembrane protease, serine 2 (TMPRSS2) are crucial for SARS-CoV-2 entry into host cells. ${ }^{7,8}$ While ACE2 is the main receptor for the spike (S) protein of both SARS-CoV and SARS-CoV-2, mediating viral attachment to target cells, TMPRSS2 cleaves protein $S$ at the $S 1 / S 2$ and the $S 2$ 'sites, allowing fusion of viral and cellular membranes. ${ }^{8}$ Both genes have been proposed to modulate susceptibility to SARSCoV, ${ }^{9,10}$ and are good candidates to mediate sex-related effects: ACE2 is located on the $\mathrm{X}$ chromosome, while TMPRSS2 expression is responsive to androgen/estrogen stimulation. ${ }^{11}$

\section{METHODS}

\section{Gene expression data}

Expression data for ACE and TMPRSS2 genes were obtained through the: 1) genotype-tissue expression (GTEx) database (https://gtexportal.org/home/), which was also used to extract quantitative trait loci (eQTLs) for the two genes (all data based on RNAseq experiments); and 2) Gene Expression Omnibus (GEO) repository (https://www.ncbi.nlm.nih.gov/geo/). In particular, two GEO datasets were extracted and analyzed: 1) GSE66499, reporting microarray data on 152 normal lung samples from Caucasian individuals; 2) GSE19804, reporting microarray data on 60 normal lung samples from Taiwanese females.

\section{Genetic data}

Genetic data for general European and East Asian populations were retrieved through the GnomAD repository, which contains data on a total of 125,748 exomes and 71,702 genomes (https://gnomad.broadinstitute.org/).

As for Italians, details on whole-exome sequencing (on 3,984 individuals) and genome-wide microarray genotyping (on 3,284 individuals) of the analyzed cohort are specified elsewhere. ${ }^{12-14}$ Imputation procedures are detailed in Supplementary materials.

\section{Statistical analysis}


Expression levels were compared by using either the Kruskal-Wallis test (RNAseq data) or the student $t$ test (microarray data). Allele frequencies were compared using the chi square test. All calculations were performed using the $R$ software (https://www.r-project.org/). P values are presented as non-corrected for multiple testing. 


\section{RESULTS AND DISCUSSION}

\section{ACE2}

For most X-chromosome genes, the double allelic dosage in women is balanced by the epigenetic silencing of one of the $X$ chromosomes in early development. ${ }^{15}$ However, the $\mathrm{X}$-chromosome inactivation $(\mathrm{XCl})$ is incomplete in humans and up to one third of genes are expressed from both alleles, with the degree of $\mathrm{XCl}$ escape varying between genes and individuals. ${ }^{16}$ ACE2 is one of the genes escaping $\mathrm{X}$ inactivation, but it belongs to a subgroup of $\mathrm{X}$-chromosome genes escaping $\mathrm{XCl}$ showing an uncharacteristically heterogeneous pattern of male-female expression, with higher expression in men in several tissues. ${ }^{17}$ Specifically concerning the lung, a recent analysis on published expression data, reported a substantial similar level of ACE2 transcript in men and women, ${ }^{18}$ however, another study, using single-cell sequencing, found a higher expression of ACE2 in Asian men. ${ }^{19}$ Supplementary Figure 1 reports data on ACE2 mRNA expression levels in the lung as retrieved from the largest datasets available in the literature; no substantial differences were found between males and females, nor between younger and older women, thus confirming what already observed by Cai and colleagues. ${ }^{18}$

Another possible sex-related effect might be due to the fact that males are hemizygous for the gene, therefore, in the presence of an ACE2 allelic variant increasing disease susceptibility or severity, men will have all cells expressing the risk variant. Based on this hypothesis, we looked into the genetic variation in ACE2. A recent manuscript explored this same topic in different populations using data from public databases. ${ }^{20}$ However, a specific analysis of the Italian population is lacking.

We have therefore exploited the available data on 3,984 exomes obtained from an Italian cohort representative of the whole country ${ }^{12,13}$ to extract the variants in exons and splice junctions of ACE2. Variants were filtered for quality and classified according to their predicted effect at protein level and on splicing. Concerning rare variants (i.e. those with a minor allele frequency, MAF, <1\%; to be used in burden tests), we considered only null variants, abolishing or significantly impairing protein production (nonsense, out-of-frame ins/dels, and splicing variants), and missense variants predicted to be deleterious or possibly deleterious by all the 5 prediction algorithms used (see Supplementary Methods). Concerning common variants (i.e., MAF> $5 \%$ ), all 
were retained for comparing their frequency with those of the European (non Finnish) and East Asian populations, retrieved from the GnomAD repository.

No significant differences in the burden of rare deleterious variants were observed comparing the Italian population with Europeans and East Asians (Table 1A). Concerning common exonic variants, the only striking difference, as also noticed by Cao and colleagues, ${ }^{20}$ was observed for the single nucleotide polymorphism (SNP) rs2285666 (also called G8790A), with the frequency of the rare A allele being 0.2 in Italians and Europeans, and 0.55 in East Asians $\left(P=2.2^{*} 10-16\right.$ for difference in Italians vs East Asians; Table 1B). This variant was extensively studied as a potential risk factor for hypertension, type 2 diabetes, and coronary artery disease, ${ }^{21,22}$ hence possibly constituting a predisposing factor also for the comorbidities observed in COVID-19 patients. A single paper reports the association of the three rs2285666 genotypes with ACE2 protein level measured in serum by ELISA, with the A/A genotype having an expression level almost $50 \%$ lower than the G/G genotype. ${ }^{23}$ Given the position of the variant, at nucleotide +4 in the donor splice site of intron 3 $(c .439+4 G>A)$, we calculated the predicted effect on splicing and indeed the substitution of $G$ with an $A$ is predicted to increase the strength of the splice site of about 9.2\% (calculation made through the Human Splicing Finder v.3.1 webtool, http://www.umd.be/HSF/), not consistently with the higher level of ACE2 protein in serum. Hence, it would be crucial to compare the frequency of this variant with ACE2 expression in the lung and with susceptibility to viral infection and severity of COVID19 manifestations. Of note, no eQTL for ACE2 in the lung has been described so far in the GTEx database, and investigations on this topic are recommended.

\section{TMPRSS2}

Our second candidate is TMPRSS2. This gene is well known to oncologists as genetic rearrangements producing a fusion between TMPRSS2 and ERG (or, more rarely, other members of the ETS family) are the most frequent genetic lesions in prostate cancer patients. ${ }^{24}$ As TMPRSS2 is an androgen responsive gene, the fusion results in androgen dependent transcription of ERG in prostate tumor cells. Therefore, we can hypothesize that men might have higher TMPRSS2 expression also in the lung, which might improve the ability of SARS-CoV-2 to enter cells by promoting membrane fusion. Looking into GTEx and GEO data, the overall expression of TMPRSS2 in the lung is only slightly increased in men $(P=0.029$; Figure $1 \mathrm{~A})$. However, TMPRSS2 expression 
is also promoted by estrogens, ${ }^{11}$ and therefore the situation might be different when considering individuals above 60 years, who are at higher risk of fatal events due to COVID-19, as in this group women will all be postmenopausal. According to this hypothesis, we checked the expression of the gene in lungs of men and females at different ages, but no substantial differences emerged between males and females (neither below, nor above 60 years of age; data not shown).

Finally, we explored genetic variation in TMPRSS2 in search of variants, possibly already annotated as eQTL in the lung, which might have an impact on the serine protease expression as well as on its catalytic activity. Again, we used the available Italian exome data, as well as data deposited in GnomAD. ${ }^{25}$

Firstly, we looked at the overall burden of deleterious rare variants, using the variant classification described above. Italians had a significant decrease in the burden of deleterious variants compared to Europeans $(P=0.039$; Table $2 A)$ suggesting that they might have a higher level of TMPRSS2 protein/activity, which in principle should be a risk factor for more severe disease course. This is even more true for the East Asian population, however, in this case, we must consider that the number of individuals over 65 years of age in Italy is more than double the one in the Hubei province (22.7 vs. $10 \%$, respectively) and this is a major determinant of disease lethality.

Focusing specifically on common exonic variants, 4 SNPs showed significantly $(P<2.2 * 10-16)$ different frequencies when comparing the Italian population with East Asians (and with Europeans) (Table 2B); 3 of them are synonymous variants, whereas one is the missense substitution p.Val160Met, which impacts on a residue far from the serine protease catalytic triad. This variant was previously found significantly associated with genomic rearrangements involving TMPRSS2, with the risk of prostate cancer $^{26}$ and with shorter time to prostate cancer diagnosis for high-risk patients. ${ }^{27}$

Concerning eQTLs, a number of variants significantly impacting on TMPRSS2 expression in the lung (GTex data) are reported in the 3 ' region of the gene (Figure 1B). In Table 2C, a list of the most significant $\left(P<1^{*} 10-8\right)$, together with their GnomAD frequencies in the East Asian and European populations, are reported. As for the Italian frequencies, we took advantage of the genome-wide association study (GWAS) performed on the above-described cohort (for a total of 3,284 individuals); $;^{14}$ in this case, we had to infer genotype frequencies by an imputation approach (for details, see Supplementary Methods). Interestingly, all these eQTLs appear to have extremely 
different frequencies among populations. In particular, 2 different haplotypes can be inferred from frequency data:

1) A frequent "European" haplotype (composed at least of SNPs rs463727, rs34624090, rs55964536, rs734056, rs4290734, rs34783969, rs11702475, rs35899679, and rs35041537), which is totally absent in the Asian population. Interestingly, this haplotype has been functionally linked to another eQTL (rs8134378), located at a known androgen-responsive enhancer for TMPRSS2, $13 \mathrm{~kb}$ upstream of the TMPRSS2 transcription start site ${ }^{28}$ (Figure 1B). Hence, this haplotype is expected to up regulate TMPRSS2 gene expression in an androgen-specific way.

2) A second haplotype, predicted to be associated with higher TMPRSS2 expression, is characterized by 3 SNPs (rs2070788, rs9974589, rs7364083), whose MAF is significantly increased in Europeans (9\% increase in Italians respect to East Asians, $P<1.9^{*} 10^{-10}$ ). Importantly, a small-scale GWAS, comparing the distribution of genetic variants in severe and mild cases of patients with $A(H 1 N 1) p d m 09$ influenza, identified rs2070788 as being associated with increased risk to both human $A(H 7 N 9)$ and severe $A(H 1 N 1) p d m 09$ influenza. ${ }^{10}$ Of note, also in $A(H 7 N 9)$ influenza, the proportion of male patients was more than double that of female patients. ${ }^{29}$

\section{Limitations and conclusions}

We are aware of the limitations of our study: first of all we focused our attention only to two candidate genes identified on the basis of their crucial role in viral infection and on the a priori probability that they might mediate sex-specific effects. A number of other X-linked genes (such as IL13, IL4, IL10, XIST, TLR7, FOXP3) and Y-linked genes (SRY, SOX9) may underlie sexually dimorphic immune responses. ${ }^{30}$ Moreover, the number of non-genetic determinants of sex-biased severity and case fatality rates is huge and probably has to do not only with sex differences in both innate and adaptive immune responses, ${ }^{6}$ but also with gender and cultural habits in different countries.

In conclusion, we have explored possible genetic components impacting on the sexbiased severity of COVID-19, focusing on effects mediated by ACE2 and TMPRSS2 genes in the Italian population. From available data, it seems unlikely that sexdifferences in ACE2 levels can explain sex differences in disease severity. However, it remains to be evaluated if changes in ACE2 levels in the lung correlate with susceptibility and severity of SARS-CoV-2 infection. Experimental data from patients 
with different disease manifestations are urgently needed. Among the analyzed hypotheses, the most interesting signals refer to sex-related differences in TMPRSS2 expression and in genetic variation in TMPRSS2. In particular, we identified an exonic variant (p.Val160Met) and 2 distinct haplotypes showing profound frequency differences between East Asians and Italians. The rare alleles of these haplotypes, all predicted to induce higher levels of TMPRSS2, are more frequent in the Italian than in the East Asian population; in one case, the haplotype could be regulated through androgens (possibly explaining the sex bias in COVID-19 severity?), in the other case, a SNP belonging to the haplotype has been associated with increased susceptibility to influenza, possibly related to a higher susceptibility in Italians and Europeans.

Our data, beside suggesting possible explanations for the unusually high, relative to known data, lethality rates among Italians, provide reference frequencies in the general Italian population for candidate variants that can be compared to genetic data from patients infected by SARS-CoV-2 with different disease manifestations, as soon as they will be available on large numbers of patients. These studies will hopefully help to identify useful prognostic markers to stratify patients and provide the best care to high-risk individuals.

\section{FUNDING}

This work was supported by Ricerca Corrente (Italian Ministry of Health), intramural funding (Fondazione Humanitas per la Ricerca). A generous contribution of the Dolce\&Gabbana Fashion Firm is gratefully acknowledged.

\section{AUTHORS' CONTRIBUTIONS}

All authors contributed to the study design. EMP did the genetic analysis, RA performed the statistical analysis, SD drafted the manuscript and supervised the entire study. All authors critically reviewed the manuscript and approved the final draft.

\section{CONFLICT OF INTEREST STATEMENTS}

No conflict of interest to disclose. 


\section{REFERENCES}

1. Zhang JJ, Dong $X$, Cao $Y$, et al. Clinical characteristics of 140 patients infected with SARS-CoV-2 in Wuhan, China. Allergy 2020; published online Feb 19. doi: $10.1111 /$ all.14238.

2. Andersen KG, Rambaut A, Lipkin WA, Holmes EC, Garry RF. The proximal origin of SARS-CoV-2. Nat Med 2020; prepublished on line March 17.

3. Jin JM, Bai P, He W, et al. Gender differences in patients with COVID-19: Focus on severity and mortality. medRxiv 2020.02.23.20026864; doi: https://doi.org/10.1101/2020.02.23.20026864.

4. Channappanavar R, Fett C, Mack M, Ten Eyck PP, Meyerholz DK, Perlman S. Sex-Based Differences in Susceptibility to Severe Acute Respiratory Syndrome Coronavirus Infection. J Immunol 2017; 198: 4046-53.

5. Alghamdi IG, Hussain II, Almalki SS, Alghamdi MS, Alghamdi MM, El-Sheemy MA. The pattern of Middle East respiratory syndrome coronavirus in Saudi Arabia: a descriptive epidemiological analysis of data from the Saudi Ministry of Health. Int J Gen Med 2014; 7: 417-23.

6. Klein SL, Flanagan KL. Sex differences in immune responses. Nat Rev Immunol 2016; 16: 626-38.

7. Zhou $P$, Yang $X L$, Wang XG, et al. A pneumonia outbreak associated with a new coronavirus of probable bat origin. Nature 2020; 579: 270-3.

8. Hoffmann M, Kleine-Weber $\mathrm{H}$, Schroeder S, et al. SARS-CoV-2 Cell Entry Depends on ACE2 and TMPRSS2 and Is Blocked by a Clinically Proven Protease Inhibitor. Cell 2020; published online Mar 4. doi: 10.1016/j.cell.2020.02.052.

9. Kuba K, Imai $\mathrm{Y}$, Rao $\mathrm{S}$, et al. A crucial role of angiotensin converting enzyme 2 (ACE2) in SARS coronavirus-induced lung injury. Nat Med 2005; 11: 875-9.

10. Cheng Z, Zhou J, To KK, et al. Identification of TMPRSS2 as a Susceptibility Gene for Severe 2009 Pandemic A(H1N1) Influenza and A(H7N9) Influenza. J Infect Dis 2015; 212: 1214-21.

11. Baena E, Shao Z, Linn DE, et al. ETV1 directs androgen metabolism and confers aggressive prostate cancer in targeted mice and patients. Genes Dev 2013; 27: 683-98.

12. Do $\mathrm{R}$, Stitziel $\mathrm{NO}$, Won $\mathrm{HH}$, et al. Exome sequencing identifies rare LDLR and APOA5 alleles conferring risk for myocardial infarction. Nature 2015; 518:102-6.

13. Paraboschi EM, Khera AV, Merlini PA, et al. Rare variants lowering the levels of coagulation factor $X$ are protective against ischemic heart disease. Haematologica 2019 Nov 7. doi: 10.3324/haematol.2019.237750. 
14. Myocardial Infarction Genetics Consortium, Kathiresan S, Voight BF, et al. Genome-wide association of early-onset myocardial infarction with single nucleotide polymorphisms and copy number variants. Nat Genet 2009; 41: 33441.

15. Lyon MF. Gene action in the X-chromosome of the mouse (Mus musculus L.). Nature 961; 190: 372-3.

16. Carrel L, Willard HF. X-inactivation profile reveals extensive variability in X-linked gene expression in females. Nature 2005; 434: 400-4.

17. Tukiainen $T$, Villani $A C$, Yen $A$, et al. Landscape of $X$ chromosome inactivation across human tissues. Nature 2017; 550: 244-8.

18. Cai G. Bulk and single-cell transcriptomics identify tobacco-use disparity in lung gene expression of ACE2, the receptor of 2019-nCov. medRxiv 2020; published online Feb 28. doi: 10.1101/2020.02.05.20020107.

19. Zhao Y, Zhao Z, Wang Y, Zhou Y, Ma Y, Zuo W. Single-cell RNA expression profiling of ACE2, the putative receptor of Wuhan 2019-nCov. bioRxiv 2020; published online Jan 26. doi: 10.1101/2020.01.26.919985.

20. Cao Y, Li L, Feng Z, et al. Comparative genetic analysis of the novel coronavirus (2019-nCoV/SARS-CoV-2) receptor ACE2 in different populations. Cell Discov 2020; 6: 11.

21. Chaoxin J, Daili S, Yanxin H, Ruwei G, Chenlong W, Yaobin T. The influence of angiotensin-converting enzyme 2 gene polymorphisms on type 2 diabetes mellitus and coronary heart disease. Eur Rev Med Pharmacol Sci 2013; 17: 2654-9.

22. Yang $M$, Zhao J, Xing L, Shi L. The association between angiotensin-converting enzyme 2 polymorphisms and essential hypertension risk: A meta-analysis involving 14,122 patients. J Renin Angiotensin Aldosterone Syst 2015; 16: 12404.

23. Wu YH, Li JY, Wang C, Zhang LM, Qiao H. The ACE2 G8790A Polymorphism: Involvement in Type 2 Diabetes Mellitus Combined with Cerebral Stroke. J Clin Lab Anal 2017; 31: e22033.

24. Kron KJ, Murison A, Zhou S, et al. TMPRSS2-ERG fusion co-opts master transcription factors and activates $\mathrm{NOTCH}$ signaling in primary prostate cancer. Nat Genet 2017; 49: 1336-45.

25. Karczewski KJ, Francioli LC, Tiao G, et al. Variation across 141,456 human exomes and genomes reveals the spectrum of loss-of-function intolerance across human protein-coding genes. bioRxiv doi: https://doi.org/10.1101/531210

26. FitzGerald LM, Agalliu I, Johnson K, et al. Association of TMPRSS2-ERG gene fusion with clinical characteristics and outcomes: results from a population-based study of prostate cancer. BMC Cancer 2008; 8: 230. 
27. Giri VN, Ruth K, Hughes L, et al. Racial differences in prediction of time to prostate cancer diagnosis in a prospective screening cohort of high-risk men: effect of TMPRSS2 Met160Val. BJU Int 2011; 107: 466-70.

28. Clinckemalie L, Spans L, Dubois V, et al. Androgen regulation of the TMPRSS2 gene and the effect of a SNP in an androgen response element. Mol Endocrinol 2013; 27: 2028-40.

29. Jernigan DB, Cox NJ. H7N9: preparing for the unexpected in influenza. Ann Rev Med 2015; 66: 361-71.

30. Ghosh S, Klein RS. Sex Drives Dimorphic Immune Responses to Viral Infections. J Immunol 2017; 198: 1782-90. 


\section{FIGURE}

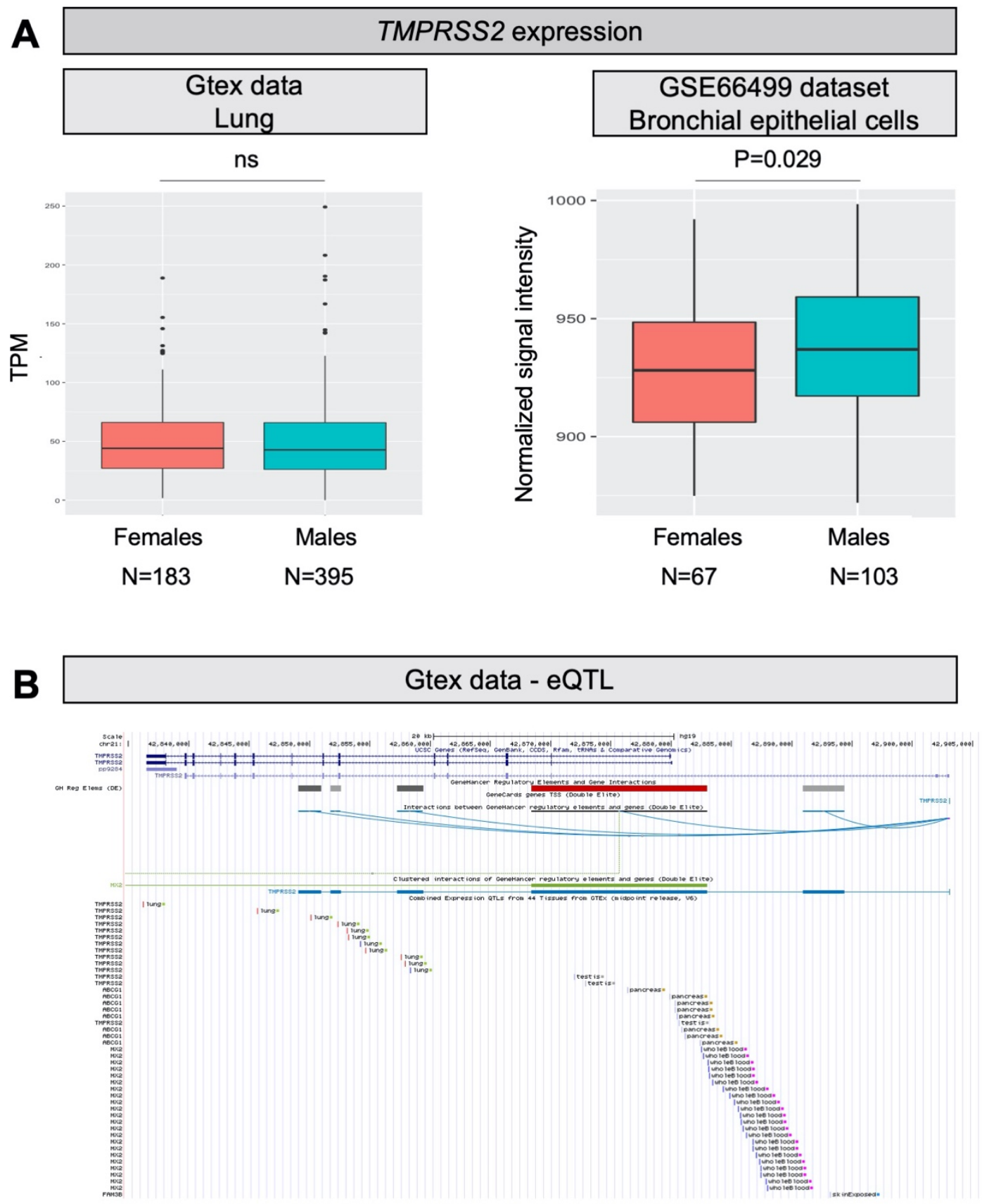

Figure 1: TMPRSS2 expression levels and eQTLs

A) Both panels show TMPRSS2 mRNA expression levels in human normal lung samples stratified according to sex. On the left, data were retrieved for a total of 578 RNAseq experiments from the GTex repository. Expression levels are reported as transcripts per kilobase million (TPM). On the right, data were collected for a total of 170 microarray experiments from the GEO database. Expression levels are reported as normalized signal intensities. $P$ values were calculated by using either the KruskalWallis or the student $t$ test. 
B) Screenshot from the UCSC Genome browser (http://genome.ucsc.edu/; GRCh37/hg19) highlighting the TMPRSS2 region (coordinates chr21: 42,835,000$42,905,000)$. The panel shows the following tracks: i) the ruler with the scale at the genomic level; ii) chromosome 21 nucleotide numbering; iii) the UCSC RefSeq track; iv) enhancers (grey and red bars) from GeneHancer database; v) interactions (curved lines) connecting GeneHancer regulatory elements and genes: all curved lines converge towards the androgen-responsive enhancer for the TMPRSS2 gene described by Clinckemalie and colleagues. ${ }^{28}$ 
Table 1A. Burden of rare mutations in the ACE2 gene in different populations.

\begin{tabular}{ccccccc}
\hline Population & N alleles & T1 & Freq T1 & ITA & EUR & EAS \\
\hline ITA & 4422 & 7 & 0.0016 & - & $\mathrm{P}=0.518$ & $\mathrm{P}=0.974$ \\
\hline EUR & 92545 & 200 & 0.0022 & $\mathrm{P}=0.518$ & - & $\mathrm{P}=0.077$ \\
\hline EAS & 14840 & 21 & 0.0014 & $\mathrm{P}=0.974$ & $\mathrm{P}=0.077$ & - \\
\hline
\end{tabular}

Total allele counts, carrier allele counts, and carrier frequencies are shown; only deleterious variants with MAF less than $1 \%$ were considered in the burden analysis. The 'deleterious' set is defined by missense variations predicted to be possibly damaging by all the 5 algorithms used (LRT score, MutationTaster, PolyPhen-2 HumDiv, PolyPhen-2 HumVar, and SIFT), and loss-of-function variants (nonsense, frameshift, and splicing variants affecting the donor/acceptor sites).

T1: alleles carrying damaging variants; Freq T1: frequency of T1 allele; ITA: Italian population; EUR: European population; EAS: East Asian population 
Table 1B. Common exon variants in the ACE2 gene in different populations.

\begin{tabular}{|c|c|c|c|c|c|c|c|c|c|c|}
\hline Variant ID & Consequence & $\begin{array}{c}\text { A1/ } \\
\text { N alleles } \\
\text { ITA }\end{array}$ & $\begin{array}{l}\text { Freq } \\
\text { ITA }\end{array}$ & $\begin{array}{c}\text { A1/ } \\
\mathrm{N} \text { alleles } \\
\text { EUR }\end{array}$ & $\begin{array}{l}\text { Freq } \\
\text { EUR }\end{array}$ & $\begin{array}{c}\text { A1/ } \\
\mathrm{N} \text { alleles } \\
\text { EAS }\end{array}$ & $\begin{array}{l}\text { Freq } \\
\text { EAS }\end{array}$ & $\begin{array}{l}\text { ITA } \\
\text { Vs } \\
\text { EUR }\end{array}$ & $\begin{array}{l}\text { ITA } \\
\text { Vs } \\
\text { EAS }\end{array}$ & $\begin{array}{c}\text { EUR } \\
\text { Vs } \\
\text { EAS }\end{array}$ \\
\hline rs2285666 & c. $439+4 G>A$ & $\begin{array}{l}909 / \\
4408\end{array}$ & 0.206 & $\begin{array}{l}17240 / \\
86164\end{array}$ & 0.200 & $\begin{array}{l}7336 / \\
13387\end{array}$ & 0.548 & 0.331 & $P<2.2 e-16$ & $P<2.2 e-16$ \\
\hline rs35803318 & p.Val749Val & $\begin{array}{l}235 / \\
4422 \\
\end{array}$ & 0.053 & $\begin{array}{l}3935 / \\
88946 \\
\end{array}$ & 0.044 & $0 / 13918$ & 0.0 & $P=0.0058$ & $P<2.2 e-16$ & $P<2.2 e-16$ \\
\hline
\end{tabular}

Total allele counts, carrier allele counts, and carrier frequencies are shown; only variants with MAF more than $5 \%$ were considered.

A1: alleles carrying variants; Freq A1: frequency of A1 allele; ITA: Italian population; EUR: European population; EAS: East Asian population 
Table 2A. Burden of rare mutations in the TMPRSS2 gene in different populations.

\begin{tabular}{ccccccc}
\hline Population & N alleles & T1 & Freq T1 & ITA & EUR & EAS \\
\hline ITA & 7968 & 30 & 0.0038 & - & $P=0.039$ & $P=3.6 E-05$ \\
\hline EUR & 129920 & 726 & 0.0056 & $P=0.039$ & - & $P=9.8 E-16$ \\
\hline EAS & 19979 & 25 & 0.0013 & $P=3.6 E-05$ & $P=9.8 E-16$ & - \\
\hline
\end{tabular}

Total allele counts, carrier allele counts, and carrier frequencies are shown; only deleterious variants with MAF less than $1 \%$ were considered in the burden analysis. The 'deleterious' set is defined by missense variations predicted to be possibly damaging by all the 5 algorithms used (LRT score, MutationTaster, PolyPhen-2 HumDiv, PolyPhen-2 HumVar, and SIFT), and loss-of-function variants (nonsense, frameshift, and splicing variants affecting the donor/acceptor sites).

T1: alleles carrying damaging variants; Freq T1: frequency of T1 allele; ITA: Italian population; EUR: European population; EAS: East Asian population 
Table 2B. Common exon variants in the TMPRSS2 gene in different populations.

\begin{tabular}{|c|c|c|c|c|c|c|c|c|c|c|}
\hline Variant ID & Consequence & $\begin{array}{c}\text { A1/ } \\
\mathrm{N} \text { alleles } \\
\text { ITA }\end{array}$ & $\begin{array}{l}\text { Freq } \\
\text { ITA }\end{array}$ & $\begin{array}{c}\mathrm{A1} / \\
\mathrm{N} \text { alleles } \\
\text { EUR }\end{array}$ & $\begin{array}{l}\text { Freq } \\
\text { EUR }\end{array}$ & $\begin{array}{c}\text { A1/ } \\
\mathrm{N} \text { alleles } \\
\text { EAS }\end{array}$ & $\begin{array}{l}\text { Freq } \\
\text { EAS }\end{array}$ & $\begin{array}{c}\text { ITA } \\
\text { Vs } \\
\text { EUR }\end{array}$ & $\begin{array}{l}\text { ITA } \\
\text { Vs } \\
\text { EAS }\end{array}$ & $\begin{array}{c}\text { EUR } \\
\text { Vs } \\
\text { EAS }\end{array}$ \\
\hline rs2298659 & p.Gly259Gly & $\begin{array}{l}1388 / \\
7968\end{array}$ & 0.174 & $\begin{array}{l}28744 / \\
122880\end{array}$ & 0.234 & $\begin{array}{l}5179 / \\
19478\end{array}$ & 0.266 & $\mathrm{P}<2.2 \mathrm{e}-16$ & $\mathrm{P}<2.2 \mathrm{e}-16$ & $\mathrm{P}<2.2 \mathrm{e}-16$ \\
\hline rs17854725 & p.lle256lle & $\begin{array}{c}4131 / \\
7968\end{array}$ & 0.518 & $\begin{array}{l}67712 / \\
122814\end{array}$ & 0.551 & $\begin{array}{l}2544 / \\
19604\end{array}$ & 0.130 & $P=1.16 e-08$ & $\mathrm{P}<2.2 \mathrm{e}-16$ & $\mathrm{P}<2.2 \mathrm{e}-16$ \\
\hline rs12329760 & p.Val160Met & $\begin{array}{l}1387 / \\
7968\end{array}$ & 0.174 & $\begin{array}{l}29831 / \\
128604\end{array}$ & 0.232 & $\begin{array}{l}7651 / \\
19934\end{array}$ & 0.384 & $P<2.2 e-16$ & $\mathrm{P}<2.2 \mathrm{e}-16$ & $P<2.2 e-16$ \\
\hline rs3787950 & p.Thr75Thr & $\begin{array}{l}889 / \\
7968\end{array}$ & 0.112 & $\begin{array}{c}9864 / \\
127666\end{array}$ & 0.077 & $\begin{array}{l}2905 / \\
19600\end{array}$ & 0.148 & $\mathrm{P}<2.2 \mathrm{e}-16$ & $P=1.39 e-15$ & $P<2.2 e-16$ \\
\hline
\end{tabular}

Total allele counts, carrier allele counts, and carrier frequencies are shown; only variants with MAF more than $5 \%$ were considered.

A1: alleles carrying variants; Freq A1: frequency of A1 allele; ITA: Italian population; EUR: European population; EAS: East Asian population. 
Table 2C. eQTL variants in the TMPRSS2 gene in different populations.

\begin{tabular}{|c|c|c|c|c|c|c|c|c|}
\hline Variant ID & $\begin{array}{c}\mathbf{P} \\
\text { GTEx }\end{array}$ & $\begin{array}{l}\text { NES } \\
\text { GTEx }\end{array}$ & $\begin{array}{c}\text { Freq } \\
\text { ITA }\end{array}$ & $\begin{array}{l}\text { Freq } \\
\text { EUR }\end{array}$ & $\begin{array}{l}\text { Freq } \\
\text { EAS }\end{array}$ & $\begin{array}{c}\text { ITA vs } \\
\text { EUR }\end{array}$ & $\begin{array}{l}\text { ITA vs } \\
\text { EAS }\end{array}$ & $\begin{array}{c}\text { EUR vs } \\
\text { EAS }\end{array}$ \\
\hline rs463727 & $5.0 e-10$ & 0.12 & 0.44 & 0.46 & 0.0051 & $P=0.038$ & $P<2.2 e-16$ & $P<2.2 e-16$ \\
\hline rs2070788 & $8.9 e-9$ & -0.11 & 0.55 & 0.53 & 0.66 & $P=0.003$ & $P=4.7 e-15$ & $P<2.2 e-16$ \\
\hline rs9974589 & $7.4 e-9$ & -0.12 & 0.55 & 0.53 & 0.66 & $P=0.002$ & $P=3.3 e-15$ & $P<2.2 e-16$ \\
\hline rs34624090 & $9.2 e-9$ & 0.12 & 0.43 & 0.45 & 0.0051 & $P=0.005$ & $P<2.2 e-16$ & $P<2.2 e-16$ \\
\hline rs7364083 & $3.3 e-9$ & -0.12 & 0.56 & 0.53 & 0.65 & $P=8.7 e-05$ & $P=1.9 e-10$ & $P<2.2 e-16$ \\
\hline rs55964536 & $1.9 e-9$ & 0.12 & 0.46 & 0.49 & 0.0045 & $P=4.6 e-04$ & $P<2.2 e-16$ & $P<2.2 e-16$ \\
\hline rs734056 & $1.3 e-9$ & 0.12 & 0.47 & 0.49 & 0.0051 & $P=0.030$ & $P<2.2 e-16$ & $P<2.2 e-16$ \\
\hline rs4290734 & $8.3 e-10$ & 0.12 & 0.47 & 0.49 & 0.0051 & $P=0.019$ & $P<2.2 e-16$ & $P<2.2 e-16$ \\
\hline rs34783969 & $3.9 e-10$ & 0.12 & 0.47 & 0.49 & 0.0051 & $P=0.027$ & $P<2.2 e-16$ & $P<2.2 e-16$ \\
\hline rs11702475 & $8.4 \mathrm{e}-10$ & 0.12 & 0.47 & 0.49 & 0.0046 & $P=0.015$ & $P<2.2 e-16$ & $P<2.2 e-16$ \\
\hline rs35899679 & $7.8 \mathrm{e}-9$ & 0.11 & 0.44 & 0.46 & 0.0051 & $P=0.004$ & $P<2.2 e-16$ & $P<2.2 e-16$ \\
\hline rs35041537 & $3.6 e-9$ & 0.12 & 0.44 & 0.47 & 0.0051 & $P=8.6 e-04$ & $\mathrm{P}<2.2 \mathrm{e}-16$ & $\mathrm{P}<2.2 \mathrm{e}-16$ \\
\hline
\end{tabular}

NES: normalized effect size; Freq: frequency of the minor allele; ITA: Italian population; EUR: European population; EAS: East Asian population 